\title{
The local well-posedness for nonlinear fourth-order Schrödinger equation with mass-critical nonlinearity and derivative
}

Cuihua Guo ${ }^{1 *}$, Shulin Sun ${ }^{2}$ and Hongping Ren ${ }^{3}$

\section{"Correspondence:}

gchzjq@sxu.edu.cn

'School of Mathematical Science,

Shanxi University, Taiyuan, Shanx 030006, China

Full list of author information is

available at the end of the article

\begin{abstract}
We study the Cauchy problem of the nonlinear fourth-order Schrödinger equation with mass-critical nonlinearity and derivative: $i u_{t}+a u_{x x x x}+b u^{2} \bar{u}_{x x}+c|u|^{8} u=0, x \in R$, $t \in R$, where $a, b$, and $c$ are real numbers. We obtain the local well-posedness for the Cauchy problem with low regularity initial value data by the Fourier restriction norm method.
\end{abstract}

Keywords: nonlinear fourth-order Schrödinger equation with derivative; Fourier restriction norm method; Cauchy problem

\section{Introduction}

In [1], Fibich et al. discussed the following fourth-order Schrödinger equation:

$$
\left\{\begin{array}{l}
i u_{t}+a \Delta^{2} u+c|u|^{\alpha} u=0, \quad x \in R^{n}, t \in R \\
u(x, 0)=u_{0}(x), \quad x \in R^{n}
\end{array}\right.
$$

They gave the sufficient conditions of the existence of solutions in the space $H^{2}\left(R^{n}\right)$. For the case $\alpha=\frac{8}{n}$, the mass is still invariant under the scaling $u(x, t) \mapsto \lambda^{-\frac{4}{\alpha}} u\left(\frac{x}{\lambda}, \frac{t}{\lambda^{4}}\right)$. We call this case mass-critical.

Meanwhile, in [1], Fibich et al. mentioned the physical motivation of (1.1). With nonparaxial effects, one obtained the perturbed nonlinear Schrödinger equation:

$$
\left\{\begin{array}{l}
i u_{t}(x, y, t)+\Delta u+\varepsilon \Delta^{2} u \\
\quad+\varepsilon\left(|u|^{2} u+|u|^{4} u+|u|^{2} \Delta u+u \nabla u \cdot \nabla \bar{u}+\bar{u} \nabla u \cdot \nabla u\right)=0 \\
u(x, y, 0)=u_{0}(x, y)
\end{array}\right.
$$

Furthermore, with vectorial effects, one obtained the following equation:

$$
\left\{\begin{array}{l}
i u_{t}(x, y, t)+\Delta u+\varepsilon \Delta^{2} u+\varepsilon\left(|u|^{2} u+|u|^{4} u+|u|^{2} \Delta u+u \nabla u \cdot \nabla \bar{u}+\bar{u} \nabla u \cdot \nabla u\right) \\
\quad+\varepsilon\left(\left|u_{x}\right|^{2} u+\left(u_{x}\right)^{2} \bar{u}+|u|^{2} u_{x x}+u^{2} \bar{u}_{x x}\right)=0, \\
u(x, y, 0)=u_{0}(x, y) .
\end{array}\right.
$$

Evidently, the nonlinearities with derivatives appear. It is well known that nonlinearities with derivatives bring about more difficulties to solve the problem for us. Especially, there are so many nonlinearities with derivatives in (1.2) and (1.3).

O2014 Guo et al.; licensee Springer. This is an Open Access article distributed under the terms of the Creative Commons Attribution License (http://creativecommons.org/licenses/by/2.0), which permits unrestricted use, distribution, and reproduction in any medium, provided the original work is properly cited. 
So, in this paper we will study the following nonlinear fourth-order Schrödinger equation with mass-critical nonlinearity and two-order derivative in one dimension:

$$
\left\{\begin{array}{l}
i u_{t}+a \Delta^{2} u+b u^{2} \bar{u}_{x x}+c|u|^{8} u=0, \quad x \in R, t \in R \\
u(x, 0)=u_{0}(x), \quad x \in R
\end{array}\right.
$$

where $u(x, t)$ are complex-valued function, $\bar{u}(x, t)$ is the complex conjugate quantity of $u(x, t) . a, b$, and $c$ are real numbers. We are interested in obtaining the well-posedness for the Cauchy problem of (1.1) with initial value data under low regularity (which means $\left.u_{0}(x) \in H^{s}(R), s<2\right)$. Tao et al. obtained the global well-posedness for the Schrödinger equations with derivative $\left(\left(|u|^{2} u\right)_{x}\right)$ by the I-method (see $\left.[2,3]\right)$. Bourgain obtained the well-posedness for the nonlinear Schrödinger equation $\left(|u|^{p} u\right)$ by the Fourier restriction norm method (see [4]). The character of (1.4) lies in the coexistence of the mass-critical nonlinearity and the two-order derivative. We will discuss the local well-posedness for the fourth-order Schrödinger equation by the Fourier restriction norm method.

For the complicated case, we will discuss it in another paper.

First, we introduce the following notations. We define the Sobolev norms $H^{s}$ by

$$
\|f\|_{H^{s}}:=\left\|\left\langle D_{x}\right\rangle^{s} f\right\|_{L_{x}^{2}}=\left\|\langle\xi\rangle^{s} \hat{f}(\xi)\right\|_{L_{\xi}^{2}}
$$

where $\langle\cdot\rangle=(1+|\cdot|)$, and $\hat{f}$ denotes the Fourier transformation of $f(x)$.

We also define the spaces $X_{s, b}(R \times R)$ (see [5]) on $R \times R$ by

$$
\|u\|_{X_{s, b}}:=\left\|\langle\xi\rangle^{s}\left\langle\tau-a \xi^{4}\right\rangle^{b} \hat{u}(\xi, \tau)\right\|_{L_{\tau}^{2} L_{\xi}^{2}}
$$

where $\hat{u}(\xi, \tau)$ denotes the Fourier transformation of $u(x, t)$.

We denote by $U(t)(t \in R)$ the fundamental solution operator of the fourth-order Schrödinger equation, i.e.,

$$
U(t) \varphi(x)=F^{-1}\left(e^{i a t \xi^{4}} \hat{\varphi}(\xi)\right) \quad \text { for } \varphi \in S^{\prime}(R),
$$

where $\hat{\varphi}$ denotes the Fourier transformation of $\varphi$, and $F^{-1}$ represents the inverse Fourier transformation.

We use $C$ to denote various constants which may be different from in particular cases of use throughout.

The main result of this paper is the following theorem.

Theorem 1.1 Let $s \geq \frac{1}{2}, \frac{1}{2}<b<\frac{5}{8}$. Then the system (1.4) is locally solvable in $H^{s}(R)$, i.e., for any $u_{0}(x) \in H^{s}(R)$, there exists a corresponding $T>0$ such that the system (1.4) has a unique solution in the class

$$
C\left([0, T] ; H^{s}(R)\right) \cap X_{s, b}
$$

Moreover, the mapping $u_{0}(x) \rightarrow u(x, t)$ is Lipschitz continuous from $H^{s}(R)$ to $C([0, T]$; $\left.H^{s}(R)\right)$. 
In [6], Cui et al. obtained the local well-posedness with the initial condition satisfying $u_{0}(x) \in H^{s}(R), s \geq 0$ for $b=0$ in (1.4).

Thus from the above theorem, we can see the following result.

Remark 1.1 When mass-critical nonlinearity and nonlinearity with derivative appear at the same time, nonlinearity with second-order derivative plays more important role. This property is consistent with the classical Schrödinger equation which has both masscritical nonlinearity and first-order derivative nonlinearity.

\section{The preliminary estimates}

Definition 2.1 For two integers $8 \leq q \leq \infty$ and $2 \leq r<\infty$, we say that $(q, r)$ is an admissible pair if the following condition is satisfied:

$$
\frac{2}{q}=\frac{1}{4}\left(1-\frac{2}{r}\right)
$$

We have the following Strichartz estimate (see [6]): For any admissible pair $(q, r)$

$$
\|U(t) \varphi(x)\|_{L_{x}^{r} L_{t}^{q}} \leq C\|\varphi\|_{L^{2}}
$$

Lemma 2.1 Assume that $(q, r)$ is an admissible pair. Let $b>\frac{1}{2}$. We have

$$
\|u\|_{L_{x}^{r} L_{t}^{q}} \leq C\|u\|_{0, b}
$$

For any $f \in L_{\tau}^{2} L_{\xi}^{2}$, we have

$$
\begin{aligned}
& \left\|D_{x}^{-\frac{1}{4}} F_{b}\right\|_{L_{x}^{4} L_{t}^{\infty}} \leq C\|f\|_{L_{\tau}^{2} L_{\xi}^{2}}, \\
& \left\|D_{x}^{\frac{3}{2}} F_{b}\right\|_{L_{x}^{\infty} L_{t}^{2}} \leq C\|f\|_{L_{\tau}^{2} L_{\xi}^{2},},
\end{aligned}
$$

where $\hat{F}_{b}(\xi, \tau)=\frac{f(\xi, \tau)}{\left(1+\mid \tau-a \xi \xi^{4}\right)^{b}}$.

Proof Firstly, we prove the inequality (2.2).

For any $u(x, t) \in S\left(R^{2}\right)$, we have

$$
u(x, t)=C \int_{R} e^{i t \lambda} \int_{R} e^{i x \xi-i t a \xi^{4}} \hat{u}\left(\xi, \lambda+a \xi^{4}\right) d \xi d \lambda=C \int_{R} e^{i t \lambda} U(t) u_{\lambda}(x) d \lambda,
$$

where $\hat{u}_{\lambda}(\xi)=\hat{u}\left(\xi, \lambda+a \xi^{4}\right)$.

Noting that $b>\frac{1}{2}$, using the Strichartz estimate, we obtain

$$
\begin{aligned}
\|u(x, t)\|_{L_{x}^{r} L_{t}^{q}} & \leq C \int_{R}\left\|U(t) u_{\lambda}(x)\right\|_{L_{x}^{r} L_{t}^{q}} d \lambda \leq C \int_{R}\left\|u_{\lambda}(x)\right\|_{L_{x}^{2}} d \lambda \\
& \leq C\left\|\langle\lambda\rangle^{b} \hat{u}_{\lambda}(\xi)\right\|_{L_{\xi}^{2} L_{\tau}^{2}}=C\|u\|_{0, b} .
\end{aligned}
$$

Next we prove (2.3), we only need to prove that for $b>\frac{1}{2}$ we have

$$
\left\|F_{b}(x, t)\right\|_{L_{x}^{4} L_{t}^{\infty}} \leq C\|f\|_{L_{\tau}^{2} \dot{H}_{\xi}^{\frac{1}{4}}} .
$$




$$
\|U(t) \varphi\|_{L_{x}^{4} L_{t}^{\infty}} \leq C\|\varphi\|_{\dot{H}^{\frac{1}{4}}} \quad \forall \varphi \in \dot{H}^{\frac{1}{4}}(R) .
$$

Changing the variable to $\tau=\lambda+a \xi^{4}$, we obtain

$$
\begin{aligned}
F_{b}(x, t) & =\int_{-\infty}^{+\infty} \int_{-\infty}^{+\infty} e^{i(x \xi+t \tau)} \frac{f(\xi, \tau)}{\left(1+\left|\tau-a \xi^{4}\right|\right)^{b}} d \xi d \tau \\
& =\int_{-\infty}^{+\infty} e^{i t \lambda}\left(\int_{-\infty}^{+\infty} e^{i x \xi+i a t \xi^{4}} f\left(\xi, \lambda+a \xi^{4}\right) d \xi\right) \frac{1}{(1+|\lambda|)^{b}} d \lambda
\end{aligned}
$$

From (2.6) and (2.7), we can obtain

$$
\begin{aligned}
\left\|F_{b}(x, t)\right\|_{L_{x}^{4} L_{t}^{\infty}} & \leq \int_{-\infty}^{+\infty} e^{i t \lambda}\left\|\int_{-\infty}^{+\infty} e^{i x \xi+i a t \xi^{4}} f\left(\xi, \lambda+a \xi^{4}\right) d \xi\right\|_{L_{x}^{4} L_{t}^{\infty}} \frac{1}{(1+|\lambda|)^{b}} d \lambda \\
& \leq C \int_{-\infty}^{+\infty}\left\|f\left(\xi, \lambda+a \xi^{4}\right)\right\|_{\dot{H}_{\xi}^{\frac{1}{4}}} \frac{1}{(1+|\lambda|)^{b}} d \lambda \\
& \leq C\|f\|_{L_{\tau}^{2} \dot{H}_{\xi}^{\frac{1}{4}}}
\end{aligned}
$$

so that (2.5) holds.

Similarly, (2.4) follows from the following inequality:

$$
\left\|D_{x}^{\frac{3}{2}} U(t) \varphi\right\|_{L_{x}^{\infty} L_{t}^{2}} \leq C\|\varphi\|_{L^{2}} .
$$

This inequality has been proved in Theorem 4.1 of [7]. We omit its detailed proof here.

Lemma 2.2 If $b>\frac{5}{8} \frac{r-2}{r}$ for $2 \leq r \leq 10$. Then we have

$$
\left\|F_{b}\right\|_{L_{x}^{r} L_{t}^{r}} \leq C\|f\|_{L_{\tau}^{2} L_{\xi}^{2}}
$$

where $F_{b}(x, t)$ is as same as in Lemma 2.1.

Proof Noting that $(10,10)$ is an admissible pair, so we have

$$
\|U(t) \varphi(x)\|_{L_{x}^{10} L_{t}^{10}} \leq C\|\varphi\|_{L^{2}(R)} .
$$

Therefore, using (2.7), Minkowski's inequality, (2.9), and taking $b>\frac{1}{2}$, we can obtain

$$
\left\|F_{b}\right\|_{L_{x}^{10} L_{t}^{10}} \leq C \int_{-\infty}^{+\infty}\left\|f\left(\xi, \lambda+a \xi^{4}\right)\right\|_{L_{\xi}^{2}} \frac{d \lambda}{(1+|\lambda|)^{b}} \leq C\|f\|_{L_{\tau}^{2} L_{\xi}^{2}}
$$

By interpolation [8] between (2.10) and the following relation:

$$
\left\|F_{0}\right\|_{L_{x}^{2} L_{t}^{2}}=\|f\|_{L_{\tau}^{2} L_{\xi}^{2}}
$$

we immediately obtain for all $b>\frac{5}{8} \frac{r-2}{r}$

$$
\left\|F_{b}\right\|_{L_{x}^{r} L_{t}^{r}} \leq C\|f\|_{L_{\tau}^{2} L_{\xi}^{2}}
$$


We take a function $\psi \in C_{0}^{\infty}(R)$ with $\psi=1$ on $[-1,1]$ and supp $\psi \subset[-2,2]$. We denote $\psi_{\delta}(\cdot)=\psi\left(\delta^{-1}(\cdot)\right)$.

Similar to the proof of Lemma 3.2 in [9] (or see [10, Lemmas 3.1-3.3], [11, Lemma 2.3], [12, Lemma 2.7]), we have the following estimates.

Lemma 2.3 For any real $s, 0<\delta<1, \frac{1}{2}<b<1$, and $\frac{1}{2}<b_{1}<b_{2}<1$. We have

$$
\begin{aligned}
& \left\|\psi_{\delta}(t) U(t) w_{0}\right\|_{X_{s, b}} \leq C \delta^{\frac{1}{2}-b}\left\|w_{0}\right\|_{H^{s}}, \\
& \left\|\psi_{\delta}(t) \int_{0}^{t} U(t-\tau) f(\tau) d \tau\right\|_{X_{s, b}} \leq C \delta^{\frac{1}{2}-b}\|f\|_{X_{s, b-1}}, \\
& \left\|\psi_{\delta}(t) F\right\|_{X_{s, b_{1}-1}} \leq C \delta^{b_{2}-b_{1}}\|F\|_{X_{s, b_{2}-1}} .
\end{aligned}
$$

Lemma 2.4 [12] Iff $f_{1}, f_{2}$, and $f_{3}$ belong to a Schwartz space on $R^{2}$, then we have

$$
\int_{\Gamma_{\tau}} \int_{\Gamma_{\xi}} \hat{\bar{f}}(\tau, \xi) \hat{f}_{1}\left(\tau_{1}, \xi_{1}\right) \hat{f}_{2}\left(\tau_{2}, \xi_{2}\right) \hat{f}_{3}\left(\tau_{3}, \xi_{3}\right) d \delta=\int_{R} \int_{R} \bar{f} f_{1} f_{2} f_{3} d x d t
$$

where

$$
\begin{aligned}
& \Gamma_{\tau}=\left\{\left(\tau_{1}, \tau_{2}, \tau_{3}\right) \in R^{3} ; \tau_{1}+\tau_{2}+\tau_{3}=\tau\right\}, \quad \Gamma_{\xi}=\left\{\left(\xi_{1}, \xi_{2}, \xi_{3}\right) \in R^{3} ; \xi_{1}+\xi_{2}+\xi_{3}=\xi\right\} \\
& d \delta=d \tau d \tau_{1} d \tau_{2} d \tau_{3} d \xi d \xi_{1} d \xi_{2} d \xi_{3} .
\end{aligned}
$$

Lemma 2.5 Let $s \geq \frac{1}{2}, \frac{1}{2}<b<\frac{11}{16}, b^{\prime}>\frac{1}{2}$. Then we have

$$
\left\|u_{1} u_{2} \bar{u}_{3 x x}\right\|_{X_{s, b-1}} \leq C\left\|u_{1}\right\|_{X_{s, b^{\prime}}}\left\|u_{2}\right\|_{X_{s, b^{\prime}}}\left\|u_{3}\right\|_{X_{s, b^{\prime}}}
$$

Proof By the definition of $X_{s, b}$ and duality, the inequality (2.11) is reduced to the following estimate:

$$
\begin{aligned}
\bigwedge & =\int_{\Gamma_{\tau}} \int_{\Gamma_{\xi}} \frac{\langle\xi\rangle^{s} i\left|\xi_{3}\right|^{2} \bar{f}(\tau, \xi) f_{1}\left(\tau_{1}, \xi_{1}\right) f_{2}\left(\tau_{2}, \xi_{2}\right) f_{3}\left(\tau_{3}, \xi_{3}\right) d \delta}{\langle\sigma\rangle^{1-b}\left\langle\xi_{1}\right\rangle^{s}\left\langle\sigma_{1}\right\rangle^{b^{\prime}}\left\langle\xi_{2}\right\rangle^{s}\left\langle\sigma_{2}\right\rangle^{b^{\prime}}\left\langle\xi_{3}\right\rangle^{s}\left\langle\sigma_{3}\right\rangle^{b^{\prime}}} \\
& \leq C\|f\|_{L_{\tau}^{2} L_{\xi}^{2}}\left\|f_{1}\right\|_{L_{\tau_{1}}^{2} L_{\xi_{1}}^{2}}\left\|f_{2}\right\|_{L_{\tau_{2}}^{2} L_{\xi_{2}}^{2}}\left\|f_{3}\right\|_{L_{\tau_{3}}^{2} L_{\xi_{3}}^{2}},
\end{aligned}
$$

for all $\bar{f} \in L^{2}$, where

$$
\begin{aligned}
& \sigma=\tau-a \xi^{4}, \quad \sigma_{1}=\tau_{1}-a \xi_{1}^{4}, \quad \sigma_{2}=\tau_{2}-a \xi_{2}^{4}, \quad \sigma_{3}=\tau_{3}+a \xi_{3}^{4}, \\
& f_{1}\left(\tau_{1}, \xi_{1}\right)=\left\langle\xi_{1}\right\rangle^{s}\left\langle\sigma_{1}\right\rangle^{b^{\prime}} \hat{u}_{1}, \quad f_{2}\left(\tau_{2}, \xi_{2}\right)=\left\langle\xi_{2}\right\rangle^{s}\left\langle\sigma_{2}\right\rangle^{b^{\prime}} \hat{u}_{2}, \quad f_{3}\left(\tau_{1}, \xi_{1}\right)=\left\langle\xi_{3}\right\rangle^{s}\left\langle\sigma_{3}\right\rangle^{b^{\prime}} \hat{\bar{u}}_{3} .
\end{aligned}
$$

Let $\hat{F}_{\rho}^{j}\left(\tau_{j}, \xi_{j}\right)=\frac{f_{j}\left(\tau_{j}, \xi_{j}\right)}{\left(1+\left|\tau_{j}-a \xi_{j}^{4}\right|\right)^{\rho}}=\frac{f_{j}\left(\tau_{j}, \xi_{j}\right)}{\left\langle\sigma_{j}\right\rangle^{\rho}}, j=1,2, \hat{F}_{\rho}^{3}\left(\tau_{3}, \xi_{3}\right)=\frac{f_{3}\left(\tau_{3}, \xi_{3}\right)}{\left(1+\left|\tau_{3}+a \xi_{3}^{4}\right|\right)^{\rho}}=\frac{f_{3}\left(\tau_{3}, \xi_{3}\right)}{\left\langle\sigma_{3}\right\rangle^{\rho}}$.

Without loss of generality, we can assume that $\bar{f} \geq 0, f_{j} \geq 0$ for $j=1,2,3$.

We split the domain of integration into two cases $|\xi| \geq 3$ and $|\xi| \leq 3$.

Case I. Assume that $|\xi| \leq 3$. 
Noting that $s \geq \frac{1}{2}$, by Lemma 2.4, the Hölder inequality, and Lemma 2.1, we obtain

$$
\begin{aligned}
\bigwedge & \leq \int_{\Gamma_{\tau}} \int_{\Gamma_{\xi}} \frac{\bar{f}(\tau, \xi) f_{1}\left(\tau_{1}, \xi_{1}\right) f_{2}\left(\tau_{2}, \xi_{2}\right)\left|\xi_{3}\right|^{\frac{3}{2}} f_{3}\left(\tau_{3}, \xi_{3}\right) d \delta}{\langle\sigma\rangle^{1-b}\left|\xi_{1}\right|^{\frac{1}{4}}\left\langle\sigma_{1}\right\rangle^{b^{\prime}}\left|\xi_{2}\right|^{\frac{1}{4}}\left\langle\sigma_{2}\right\rangle^{b^{\prime}}\left\langle\sigma_{3}\right\rangle^{b^{\prime}}} \\
& \leq C \int_{R} \int_{R}\left|\bar{F}_{0}\right| \cdot\left|D_{x}^{-\frac{1}{4}} F_{b^{\prime}}^{1}\right| \cdot\left|D_{x}^{-\frac{1}{4}} F_{b^{\prime}}^{2}\right| \cdot\left|D_{x}^{\frac{3}{2}} F_{b^{\prime}}^{3}\right| d x d t \\
& \leq C\left\|F_{0}\right\|_{L_{x}^{2} L_{t}^{2}}\left\|D_{x}^{-\frac{1}{4}} F_{b^{\prime}}^{1}\right\|_{L_{x}^{4} L_{t}^{\infty}}\left\|D_{x}^{-\frac{1}{4}} F_{b^{\prime}}^{2}\right\|_{L_{x}^{4} L_{t}^{\infty}}\left\|D_{x}^{\frac{3}{2}} F_{b^{\prime}}^{3}\right\|_{L_{x}^{\infty} L_{t}^{2}} \\
& \leq C\|f\|_{L_{\tau}^{2} L_{\xi}^{2}}\left\|f_{1}\right\|_{L_{\tau_{1}}^{2} L_{\xi_{1}}^{2}}\left\|f_{2}\right\|_{L_{\tau_{2}}^{2} L_{\xi_{2}}^{2}}\left\|f_{3}\right\|_{L_{\tau_{3}}^{2} L_{\xi_{3}}^{2}} .
\end{aligned}
$$

Case II. Assume that $|\xi| \geq 3$.

Subcase 1. Assume that $\left|\xi_{3}\right| \leq 1$. Then we have $|\xi| \leq 3 \max \left(\left|\xi_{1}\right|,\left|\xi_{2}\right|\right)$.

Noting that $1-b>\frac{5}{16}$, by Lemma 2.4 , the Hölder inequality, and Lemma 2.2, we obtain

$$
\begin{aligned}
\bigwedge & \leq C \int_{\Gamma_{\tau}} \int_{\Gamma_{\xi}} \frac{\bar{f}(\tau, \xi) f_{1}\left(\tau_{1}, \xi_{1}\right) f_{2}\left(\tau_{2}, \xi_{2}\right) f_{3}\left(\tau_{3}, \xi_{3}\right) d \delta}{\langle\sigma\rangle^{1-b}\left\langle\sigma_{1}\right\rangle^{b^{\prime}}\left\langle\sigma_{2}\right\rangle^{b^{\prime}}\left\langle\sigma_{3}\right\rangle^{b^{\prime}}} \\
& \leq C \int_{R} \int_{R}\left|\bar{F}_{1-b}\right| \cdot\left|F_{b^{\prime}}^{1}\right| \cdot\left|F_{b^{\prime}}^{2}\right| \cdot\left|F_{b^{\prime}}^{2}\right| d x d t \\
& \leq C\left\|F_{1-b}\right\|_{L_{x}^{4} L_{t}^{4}}\left\|F_{b^{\prime}}^{1}\right\|_{L_{x}^{4} L_{t}^{4}}\left\|F_{b^{\prime}}^{2}\right\|_{L_{x}^{4} L_{t}^{4}}\left\|F_{b^{\prime}}^{3}\right\|_{L_{x}^{4} L_{t}^{4}} \\
& \leq C\|f\|_{L_{\tau}^{2} L_{\xi}^{2}}\left\|f_{1}\right\|_{L_{\tau_{1}}^{2} L_{\xi_{1}}^{2}}\left\|f_{2}\right\|_{L_{\tau_{2}}^{2} L_{\xi_{2}}^{2}}\left\|f_{3}\right\|_{L_{\tau_{3}}^{2} L_{\xi_{3}}^{2}} \cdot
\end{aligned}
$$

Subcase 2. Assume that $\left|\xi_{3}\right| \geq 1$. We split this domain of integration in several pieces.

$1^{\circ}$ Assume that $|\xi| \leq 3 \max \left(\left|\xi_{1}\right|,\left|\xi_{2}\right|,\left|\xi_{3}\right|\right)=3\left|\xi_{1}\right|$.

Similar to the proof of subcase 1 , noting that $s \geq \frac{1}{2}, 1-b>\frac{5}{16}$, by Lemma 2.4, the Hölder inequality, and Lemma 2.2, we obtain

$$
\begin{aligned}
\bigwedge & \leq C \int_{\Gamma_{\tau}} \int_{\Gamma_{\xi}} \frac{\bar{f}(\tau, \xi) f_{1}\left(\tau_{1}, \xi_{1}\right) f_{2}\left(\tau_{2}, \xi_{2}\right) f_{3}\left(\tau_{3}, \xi_{3}\right) d \delta}{\langle\sigma\rangle^{1-b}\left\langle\sigma_{1}\right\rangle^{b^{\prime}}\left\langle\sigma_{2}\right\rangle^{b^{\prime}}\left\langle\sigma_{3}\right\rangle^{b^{\prime}}} \\
& \leq C\|f\|_{L_{\tau}^{2} L_{\xi}^{2}}\left\|f_{1}\right\|_{L_{\tau_{1}}^{2} L_{\xi_{1}}^{2}}\left\|f_{2}\right\|_{L_{\tau_{2}}^{2} L_{\xi_{2}}^{2}}\left\|f_{3}\right\|_{L_{\tau_{3}}^{2} L_{\xi_{3}}^{2}} .
\end{aligned}
$$

$\mathbf{2}^{\circ}$ Assume that $|\xi| \leq 3 \max \left(\left|\xi_{1}\right|,\left|\xi_{2}\right|,\left|\xi_{3}\right|\right)=3\left|\xi_{2}\right|$.

In this case, the proof is similar to that of the above case, so here we omit the detailed proof.

$3^{\circ}$ Assume that $|\xi| \leq 3 \max \left(\left|\xi_{1}\right|,\left|\xi_{2}\right|,\left|\xi_{3}\right|\right)=3\left|\xi_{3}\right|$.

In this case, we easily get

$$
|\xi| \sim\left|\xi_{3}\right|
$$

By a straightforward calculation, we can obtain

$$
\begin{aligned}
4 \max \left(|\sigma|,\left|\sigma_{1}\right|,\left|\sigma_{2}\right|,\left|\sigma_{3}\right|\right) & \geq|\sigma|+|\sigma|+\left|\sigma_{2}\right|+\left|\sigma_{3}\right| \geq\left|\sigma-\sigma_{1}-\sigma_{2}-\sigma_{3}\right| \\
& =2|a|\left|\xi \xi_{3}+\xi_{1} \xi_{2}\right|\left|2 \xi_{1}^{2}+3 \xi_{1} \xi_{2}+2 \xi_{2}^{2}+\xi \xi_{3}\right| \\
& \geq C\langle\xi\rangle^{2}\left\langle\xi_{3}\right\rangle^{2} .
\end{aligned}
$$


3.0: Assume that $|\sigma|=\max \left(|\sigma|,\left|\sigma_{1}\right|,\left|\sigma_{2}\right|,\left|\sigma_{3}\right|\right)$, so $\langle\sigma\rangle \geq C\langle\xi\rangle^{4}$ holds.

Noting that $b<\frac{11}{16}$, by Lemma 2.4, the Hölder inequality, and Lemma 2.1, we obtain

$$
\begin{aligned}
& \bigwedge=\int_{\Gamma_{\tau}} \int_{\Gamma_{\xi}} \frac{\langle\xi\rangle^{s} i\left|\xi_{3}\right|^{2} \bar{f}(\tau, \xi) f_{1}\left(\tau_{1}, \xi_{1}\right) f_{2}\left(\tau_{2}, \xi_{2}\right) f_{3}\left(\tau_{3}, \xi_{3}\right) d \delta}{\langle\sigma\rangle^{1-b}\left\langle\xi_{1}\right\rangle^{s}\left\langle\sigma_{1}\right\rangle^{b^{\prime}}\left\langle\xi_{2}\right\rangle^{s}\left\langle\sigma_{2}\right\rangle^{b^{\prime}}\left\langle\xi_{3}\right\rangle^{s}\left\langle\sigma_{3}\right\rangle^{b^{\prime}}} \\
& \leq C \int_{\Gamma_{\tau}} \int_{\Gamma_{\xi}} \frac{\langle\xi\rangle^{s} \bar{f}(\tau, \xi) f_{1}\left(\tau_{1}, \xi_{1}\right) f_{2}\left(\tau_{2}, \xi_{2}\right)\left|\xi_{3}\right|^{2} f_{3}\left(\tau_{3}, \xi_{3}\right) d \delta}{\langle\xi\rangle^{4(1-b)}\left\langle\xi_{1}\right\rangle^{s}\left\langle\sigma_{1}\right\rangle^{b^{\prime}}\left\langle\xi_{2}\right\rangle^{s}\left\langle\sigma_{2}\right\rangle^{b^{\prime}}\left\langle\xi_{3}\right\rangle^{s}\left\langle\sigma_{3}\right\rangle^{b^{\prime}}} \\
& \leq C \int_{\Gamma_{\tau}} \int_{\Gamma_{\xi}} \frac{\langle\xi\rangle^{s} \bar{f}(\tau, \xi)\left|\xi_{1}\right|^{\frac{1}{4}} f_{1}\left(\tau_{1}, \xi_{1}\right)\left|\xi_{2}\right|^{\frac{1}{4}} f_{2}\left(\tau_{2}, \xi_{2}\right)\left|\xi_{3}\right|^{\frac{3}{2}}\left|\xi_{3}\right|^{\frac{1}{2}} f_{3}\left(\tau_{3}, \xi_{3}\right) d \delta}{\langle\xi\rangle^{4(1-b)}\left\langle\xi_{1}\right\rangle^{s}\left|\xi_{1}\right|^{\frac{1}{4}}\left\langle\sigma_{1}\right\rangle^{b^{\prime}}\left|\xi_{2}\right|^{\frac{1}{4}}\left\langle\xi_{2}\right\rangle^{s}\left\langle\sigma_{2}\right\rangle^{b^{\prime}}\left\langle\xi_{3}\right\rangle^{s}\left\langle\sigma_{3}\right\rangle^{b^{\prime}}} \\
& \leq C \int_{\Gamma_{\tau}} \int_{\Gamma_{\xi}} \frac{\bar{f}(\tau, \xi) f_{1}\left(\tau_{1}, \xi_{1}\right) f_{2}\left(\tau_{2}, \xi_{2}\right)\left|\xi_{3}\right|^{\frac{3}{2}} f_{3}\left(\tau_{3}, \xi_{3}\right) d \delta}{\left|\xi_{1}\right|^{\frac{1}{4}}\left\langle\sigma_{1}\right\rangle^{b^{\prime}}\left|\xi_{2}\right|^{\frac{1}{4}}\left\langle\sigma_{2}\right\rangle^{b^{\prime}}\left\langle\sigma_{3}\right\rangle^{b^{\prime}}} \\
& \leq C \int_{R} \int_{R}\left|F_{0}\right| \cdot\left|D_{x}^{-\frac{1}{4}} F_{b^{\prime}}^{1}\right| \cdot\left|D_{x}^{-\frac{1}{4}} F_{b^{\prime}}^{2}\right| \cdot\left|D_{x}^{\frac{3}{2}} F_{b^{\prime}}^{3}\right| d x d t \\
& \leq C\left\|F_{0}\right\|_{L_{x}^{2} L_{t}^{2}}\left\|D_{x}^{-\frac{1}{4}} F_{b^{\prime}}^{1}\right\|_{L_{x}^{4} L_{t}^{\infty}}\left\|D_{x}^{-\frac{1}{4}} F_{b^{\prime}}^{2}\right\|_{L_{x}^{4} L_{t}^{\infty}}\left\|D_{x}^{\frac{3}{2}} F_{b^{\prime}}^{3}\right\|_{L_{x}^{\infty} L_{t}^{2}} \\
& \leq C\|f\|_{L_{\tau}^{2} L_{\xi}^{2}}\left\|f_{1}\right\|_{L_{\tau_{1}}^{2} L_{\xi_{1}}^{2}}\left\|f_{2}\right\|_{L_{\tau_{2}}^{2} L_{\xi_{2}}^{2}}\left\|f_{3}\right\|_{L_{\tau_{3}}^{2} L_{\xi_{3}}^{2}} .
\end{aligned}
$$

3.1: Assume that $\left|\sigma_{1}\right|=\max \left(|\sigma|,\left|\sigma_{1}\right|,\left|\sigma_{2}\right|,\left|\sigma_{3}\right|\right)$. By (2.10) and (2.11), we immediately obtain $\left\langle\sigma_{1}\right\rangle \geq C\left\langle\xi_{3}\right\rangle^{4}$.

Noting that $\frac{1}{2}<b<\frac{11}{16}, b^{\prime}>\frac{1}{2}$, by Lemma 2.4, the Hölder inequality, and Lemma 2.1, we obtain

$$
\begin{aligned}
& \bigwedge=\int_{\Gamma_{\tau}} \int_{\Gamma_{\xi}} \frac{\langle\xi\rangle^{s} i\left|\xi_{3}\right|^{2} \bar{f}(\tau, \xi) f_{1}\left(\tau_{1}, \xi_{1}\right) f_{2}\left(\tau_{2}, \xi_{2}\right) f_{3}\left(\tau_{3}, \xi_{3}\right) d \delta}{\langle\sigma\rangle^{1-b}\left\langle\xi_{1}\right\rangle^{s}\left\langle\sigma_{1}\right\rangle^{b^{\prime}}\left\langle\xi_{2}\right\rangle^{s}\left\langle\sigma_{2}\right\rangle^{b^{\prime}}\left\langle\xi_{3}\right\rangle^{s}\left\langle\sigma_{3}\right\rangle^{b^{\prime}}} \\
& \leq \int_{\Gamma_{\tau}} \int_{\Gamma_{\xi}} \frac{\langle\xi\rangle^{s} \bar{f}(\tau, \xi) f_{1}\left(\tau_{1}, \xi_{1}\right) f_{2}\left(\tau_{2}, \xi_{2}\right)\left|\xi_{3}\right|^{2} f_{3}\left(\tau_{3}, \xi_{3}\right) d \delta}{\langle\sigma\rangle^{b^{\prime}}\left\langle\xi_{1}\right\rangle^{s}\left\langle\sigma_{1}\right\rangle^{1-b}\left\langle\xi_{2}\right\rangle^{s}\left\langle\sigma_{2}\right\rangle^{b^{\prime}}\left\langle\xi_{3}\right\rangle^{s}\left\langle\sigma_{3}\right\rangle^{1-b}} \\
& \leq C \int_{\Gamma_{\tau}} \int_{\Gamma_{\xi}} \frac{\langle\xi\rangle^{s} \bar{f}(\tau, \xi) f_{1}\left(\tau_{1}, \xi_{1}\right) f_{2}\left(\tau_{2}, \xi_{2}\right)\left|\xi_{3}\right|^{2} f_{3}\left(\tau_{3}, \xi_{3}\right) d \delta}{\langle\sigma\rangle^{b^{\prime}}\left\langle\xi_{1}\right\rangle^{s}\left\langle\xi_{3}\right\rangle^{4(1-b)}\left\langle\xi_{2}\right\rangle^{s}\left\langle\sigma_{2}\right\rangle^{b^{\prime}}\left\langle\xi_{3}\right\rangle^{s}\left\langle\sigma_{3}\right\rangle^{b^{\prime}}} \\
& \leq C \int_{\Gamma_{\tau}} \int_{\Gamma_{\xi}} \frac{\left.|\xi|^{\frac{3}{2}}\langle\xi\rangle^{s} \bar{f}(\tau, \xi) f_{1}\left(\tau_{1}, \xi_{1}\right)\left|\xi_{2}\right|^{\frac{1}{4}} f_{2}\left(\tau_{2}, \xi_{2}\right)\left|\xi_{3}\right|^{2} \xi_{3}\right|^{\frac{1}{4}} f_{3}\left(\tau_{3}, \xi_{3}\right) d \delta}{|\xi|^{\frac{3}{2}}\langle\sigma\rangle^{b^{\prime}}\left\langle\xi_{1}\right\rangle^{s}\left\langle\sigma_{1}\right\rangle^{b^{\prime}}\left\langle\xi_{2}\right\rangle^{s}\left|\xi_{2}\right|^{\frac{1}{4}}\left\langle\sigma_{2}\right\rangle^{b^{\prime}}\left\langle\xi_{3}\right\rangle^{s}\left|\xi_{3}\right|^{\frac{1}{4}}\left\langle\sigma_{3}\right\rangle^{b^{\prime}}} \\
& \leq C \int_{\Gamma_{\tau}} \int_{\Gamma_{\xi}} \frac{|\xi|^{\frac{3}{2}} \bar{f}(\tau, \xi) f_{1}\left(\tau_{1}, \xi_{1}\right)\left|\xi_{2}\right|^{\frac{1}{4}} f_{2}\left(\tau_{2}, \xi_{2}\right)\left|\xi_{3}\right|^{\frac{1}{4}} f_{3}\left(\tau_{3}, \xi_{3}\right) d \delta}{\langle\sigma\rangle^{b^{\prime}}\left|\xi_{2}\right|^{\frac{1}{4}}\left\langle\sigma_{2}\right\rangle^{b^{\prime}}\left|\xi_{3}\right|^{\frac{1}{4}}\left\langle\sigma_{3}\right\rangle^{b^{\prime}}} \\
& \leq C \int_{R} \int_{R}\left|D_{x}^{\frac{3}{2}} F_{b^{\prime}}\right| \cdot\left|F_{0}^{1}\right| \cdot\left|D_{x}^{-\frac{1}{4}} F_{b^{\prime}}^{2}\right| \cdot\left|D_{x}^{-\frac{1}{4}} F_{b^{\prime}}^{3}\right| d x d t \\
& \leq C\left\|D_{x}^{\frac{3}{2}} F_{b^{\prime}}\right\|_{L_{x}^{\infty} L_{t}^{2}}\left\|F_{0}^{1}\right\|_{L_{x}^{2} L_{t}^{2}}\left\|D_{x}^{-\frac{1}{4}} F_{b^{\prime}}^{2}\right\|_{L_{x}^{4} L_{t}^{\infty}}\left\|D_{x}^{-\frac{1}{4}} F_{b^{\prime}}^{3}\right\|_{L_{x}^{4} L_{t}^{\infty}} \\
& \leq C\|f\|_{L_{\tau}^{2} L_{\xi}^{2}}\left\|f_{1}\right\|_{L_{\tau_{1}}^{2} L_{\xi_{1}}^{2}}\left\|f_{2}\right\|_{L_{\tau_{2}}^{2} L_{\xi_{2}}^{2}}\left\|f_{3}\right\|_{L_{\tau_{3}}^{2} L_{\xi_{3}}^{2}} .
\end{aligned}
$$

3.2: Assume that $\left|\sigma_{2}\right|=\max \left(|\sigma|,\left|\sigma_{1}\right|,\left|\sigma_{2}\right|,\left|\sigma_{3}\right|\right)$.

The proof is similar to that of the case 3.1, so here omit the detailed proof.

3.3: Assume that $\left|\sigma_{3}\right|=\max \left(|\sigma|,\left|\sigma_{1}\right|,\left|\sigma_{2}\right|,\left|\sigma_{3}\right|\right)$. By (2.10) and (2.11), we immediately obtain $\left\langle\sigma_{3}\right\rangle \geq C\left\langle\xi_{3}\right\rangle^{4}$. 
Noting that $\frac{1}{2}<b<\frac{11}{16}, b^{\prime}>\frac{1}{2}$, by Lemma 2.4, the Hölder inequality, and Lemma 2.1, we obtain

$$
\begin{aligned}
& \bigwedge=\int_{\Gamma_{\tau}} \int_{\Gamma_{\xi}} \frac{\langle\xi\rangle^{s} i\left|\xi_{3}\right|^{2} \bar{f}(\tau, \xi) f_{1}\left(\tau_{1}, \xi_{1}\right) f_{2}\left(\tau_{2}, \xi_{2}\right) f_{3}\left(\tau_{3}, \xi_{3}\right) d \delta}{\langle\sigma\rangle^{1-b}\left\langle\xi_{1}\right\rangle^{s}\left\langle\sigma_{1}\right\rangle^{b^{\prime}}\left\langle\xi_{2}\right\rangle^{s}\left\langle\sigma_{2}\right\rangle^{b^{\prime}}\left\langle\xi_{3}\right\rangle^{s}\left\langle\sigma_{3}\right\rangle^{b^{\prime}}} \\
& \leq \int_{\Gamma_{\tau}} \int_{\Gamma_{\xi}} \frac{\langle\xi\rangle^{s} \bar{f}(\tau, \xi) f_{1}\left(\tau_{1}, \xi_{1}\right) f_{2}\left(\tau_{2}, \xi_{2}\right)\left|\xi_{3}\right|^{2} f_{3}\left(\tau_{3}, \xi_{3}\right) d \delta}{\langle\sigma\rangle^{b^{\prime}}\left\langle\xi_{1}\right\rangle^{s}\left\langle\sigma_{1}\right\rangle^{b^{\prime}}\left\langle\xi_{2}\right\rangle^{s}\left\langle\sigma_{2}\right\rangle^{b^{\prime}}\left\langle\xi_{3}\right\rangle^{s}\left\langle\sigma_{3}\right\rangle^{1-b}} \\
& \leq C \int_{\Gamma_{\tau}} \int_{\Gamma_{\xi}} \frac{\langle\xi\rangle^{s} \bar{f}(\tau, \xi) f_{1}\left(\tau_{1}, \xi_{1}\right) f_{2}\left(\tau_{2}, \xi_{2}\right)\left|\xi_{3}\right|^{2} f_{3}\left(\tau_{3}, \xi_{3}\right) d \delta}{\langle\sigma\rangle^{b^{\prime}}\left\langle\xi_{1}\right\rangle^{s}\left\langle\sigma_{1}\right\rangle^{b^{\prime}}\left\langle\xi_{2}\right\rangle^{s}\left\langle\sigma_{2}\right\rangle^{b^{\prime}}\left\langle\xi_{3}\right\rangle^{s}\left\langle\xi_{3}\right\rangle^{4(1-b)}} \\
& \leq C \int_{\Gamma_{\tau}} \int_{\Gamma_{\xi}} \frac{\left\langle|\xi|^{\frac{3}{2}} \xi\right\rangle^{s} \bar{f}(\tau, \xi)\left|\xi_{1}\right|^{\frac{1}{4}} f_{1}\left(\tau_{1}, \xi_{1}\right)\left|\xi_{2}\right|^{\frac{1}{4}} f_{2}\left(\tau_{2}, \xi_{2}\right)\left|\xi_{3}\right|^{2} f_{3}\left(\tau_{3}, \xi_{3}\right) d \delta}{|\sigma\rangle^{b^{\prime}}\left|\xi_{1}\right|^{\frac{1}{4}}\left\langle\xi_{1}\right\rangle^{s}\left\langle\sigma_{1}\right\rangle^{b^{\prime}}\left|\xi_{2}\right|^{\frac{1}{4}}\left\langle\xi_{2}\right\rangle^{s}\left\langle\sigma_{2}\right\rangle^{b^{\prime}}\left\langle\xi_{3}\right\rangle^{s}\left\langle\xi_{3}\right\rangle^{4(1-b)}} \\
& \leq C \int_{\Gamma_{\tau}} \int_{\Gamma_{\xi}} \frac{|\xi|^{\frac{3}{2}} \bar{f}(\tau, \xi) f_{1}\left(\tau_{1}, \xi_{1}\right) f_{2}\left(\tau_{2}, \xi_{2}\right) f_{3}\left(\tau_{3}, \xi_{3}\right) d \delta}{\langle\sigma\rangle^{b^{\prime}}\left|\xi_{1}\right|^{\frac{1}{4}}\left\langle\sigma_{1}\right\rangle^{b^{\prime}}\left|\xi_{2}\right|^{\frac{1}{4}}\left\langle\sigma_{2}\right\rangle^{b^{\prime}}} \\
& \leq C \int_{R} \int_{R}\left|D_{x}^{\frac{3}{2}} F_{b^{\prime}}\right| \cdot\left|D_{x}^{-\frac{1}{4}} F_{b^{\prime}}^{1}\right| \cdot\left|D_{x}^{-\frac{1}{4}} F_{b^{\prime}}^{2}\right| \cdot\left|F_{0}^{3}\right| d x d t \\
& \leq C\left\|D_{x}^{\frac{3}{2}} F_{b^{\prime}}\right\|_{L_{x}^{\infty} L_{t}^{2}}\left\|D_{x}^{-\frac{1}{4}} F_{b^{\prime}}^{1}\right\|_{L_{x}^{4} L_{t}^{\infty}}\left\|D_{x}^{-\frac{1}{4}} F_{b^{\prime}}^{2}\right\|_{L_{x}^{4} L_{t}^{\infty}}\left\|F_{0}^{3}\right\|_{L_{x}^{2} L_{t}^{2}} \\
& \leq C\|f\|_{L_{\tau}^{2} L_{\xi}^{2}}\left\|f_{1}\right\|_{L_{\tau_{1}}^{2} L_{\xi_{1}}^{2}}\left\|f_{2}\right\|_{L_{\tau_{2}}^{2} L_{\xi_{2}}^{2}}\left\|f_{3}\right\|_{L_{\tau_{3}}^{2} L_{\xi_{3}}^{2}} .
\end{aligned}
$$

Lemma 2.6 Let $s \geq \frac{1}{2}, \frac{1}{2}<b<\frac{5}{8}$. Then we have the following inequality:

$$
\left\|\prod_{j=1}^{9} u_{j}\right\|_{X_{s, b-1}} \leq C \prod_{j=1}^{9}\left\|u_{j}\right\|_{X_{s, b}} .
$$

Proof Firstly, we prove (2.14) holds for $s=0$.

By the definition, the Hölder inequality, and the Sobolev inequality, we have

$$
\begin{aligned}
\|u\|_{0, b-1} & =\sup _{\|v\|_{0,1-b} \leq 1}|\langle u, v\rangle| \leq \sup _{\|v\|_{0,1-b} \leq 1}\|u\|_{L_{x}^{2} L_{t}^{3-2 b}}\|v\|_{L_{x}^{2} L_{t}^{\frac{2}{2 b-1}}} \\
& \leq C \sup _{\|v\|_{0,1-b} \leq 1}\|u\|_{L_{x}^{2} L_{t}^{3-2 b}}\|v\|_{L_{x}^{2} \dot{W}_{t}^{1-b, 2} \leq C} \sup _{\|v\|_{0,1-b} \leq 1}\|u\|_{L_{x}^{2} L_{t}^{\frac{2}{3-2 b}}}\|v\|_{0,1-b} \\
& \leq C\|u\|_{L_{x}^{2} L_{t}^{\frac{2}{3-2 b}}}
\end{aligned}
$$

That means

$$
\left\|\prod_{j=1}^{9} u_{j}\right\|_{0, b-1} \leq C\left\|\prod_{j=1}^{9} u_{j}\right\|_{L_{x}^{2} L_{t}^{\frac{2}{3-2 b}}} \cdot
$$

Again using the Hölder inequality, the Sobolev inequality in the variable $x$, and Lemma 2.1, we obtain

$$
\left\|\prod_{j=1}^{9} u_{j}\right\|_{L_{x}^{2} L_{t}^{\frac{2}{3-2 b}}} \leq \prod_{j=1}^{9}\left\|u_{j}\right\|_{L_{x}^{\overline{p_{j}}} L_{t}^{q_{j}}} \leq C \prod_{j=1}^{9}\left\|u_{j}\right\|_{\dot{W}_{x}^{s}{ }_{x}, p_{j}} L_{t}^{q_{j}} \leq C \prod_{j=1}^{9}\left\|u_{j}\right\|_{s_{j}, b}
$$

where $\sum_{j=1}^{9} \frac{1}{q_{j}}=\frac{3}{2}-b, \sum_{j=1}^{9} \frac{1}{\overline{p_{j}}}=\frac{1}{2}, \frac{1}{p_{j}}-\frac{1}{\overline{p_{j}}}=s_{j}, \sum_{j=1}^{9} s_{j}=4 b-2,\left(q_{j}, p_{j}\right)$ is admissible pair. 
Combining (2.16) and (2.17), we obtain

$$
\left\|\prod_{j=1}^{9} u_{j}\right\|_{0, b-1} \leq C \prod_{j=1}^{9}\left\|u_{j}\right\|_{s j}, b
$$

Secondly, using the Leibniz rule for fractional power, in a similar way, we obtain

$$
\begin{aligned}
&\left\|D_{x}^{s}\left(\prod_{j=1}^{9} u_{j}\right)\right\|_{0, b-1} \leq C \sum_{k=1}^{9} \prod_{j=1}^{9}\left\|u_{j}\right\|_{L_{x}^{k}} L_{L_{t}^{k}} \leq C \sum_{k=1}^{9} \prod_{j=1}^{9}\left\|u_{j}\right\|_{\dot{W}_{x}^{j} s_{j}^{k}, p_{j}^{k}} q_{L_{t}^{k}}^{q_{j}^{k}} \\
& \leq C \sum_{k=1}^{9} \prod_{j=1}^{9}\left\|u_{j}\right\|_{s_{j}^{k}, b},
\end{aligned}
$$

where $\sum_{j=1}^{9} \frac{1}{q_{j}^{k}}=\frac{3}{2}-b, \sum_{j=1}^{9} \frac{1}{p_{j}^{k}}=\frac{1}{2}, \frac{1}{p_{j}^{k}}-\frac{1}{\overline{p_{j}^{k}}}=s_{j}, \sum_{j=1}^{9} s_{j}^{k}=s+4 b-2,\left(q_{j}^{k}, p_{j}^{k}\right)$ is admissible pair for $k=1, \ldots, 9$.

Taking $s_{k}^{k}=s, p_{k}^{k}=\infty, q_{k}^{k}=8$; and for some $k_{0} \neq k, s_{k_{0}}^{k}=4 b-2, p_{k_{0}}^{k}=\frac{1}{4 b-2}, q_{k_{0}}^{k}=\frac{8}{5-8 b}$; $s_{j}^{k}=0, p_{j}^{k}=14, q_{j}^{k}=\frac{28}{3}$, for $j \neq k, k_{0}$ in (2.19), noting $s \geq \frac{1}{2}$ and $\frac{1}{2}<b<\frac{5}{8}$, we obtain

$$
\begin{aligned}
\left\|D_{x}^{s}\left(\prod_{j=1}^{9} u_{j}\right)\right\|_{0, b-1} & \leq C \sum_{k=1}^{9}\left\|u_{k}\right\|_{s, b}\left\|u_{k_{0}}\right\|_{4 b-2, b} \prod_{j=1, j \neq k, k_{0}}^{9}\left\|u_{j}\right\|_{0, b} \\
& \leq C \sum_{k=1}^{9}\left\|u_{k}\right\|_{s, b}\left\|u_{k_{0}}\right\|_{s, b} \prod_{j=1, j \neq k, k_{0}}^{9}\left\|u_{j}\right\|_{0, b} \\
& \leq C \prod_{j=1}^{9}\left\|u_{j}\right\|_{s, b},
\end{aligned}
$$

which completes the proof.

\section{Proof of main result}

Proof of Theorem 1.1 We will prove Theorem 1.1 by using the Banach fixed point theorem. Let $T<\delta$. We rewrite (1.4) in integral form:

$$
u(t)=U(t) u_{0}-\int_{0}^{t} U\left(t-t^{\prime}\right)\left(b u^{2} \bar{u}_{x x}+c|u|^{8} u\right)\left(t^{\prime}\right) d t^{\prime}
$$

We denote

$$
Z=\left\{u \in X_{s, b}:\|u\|_{X_{s, b}} \leq 2 C_{0}\left\|u_{0}\right\|_{H^{s}}\right\}
$$

and define a mapping $S$ as follows: For $u \in X_{s, b}$,

$$
S u(t)=\psi(t) U(t) u_{0}-\psi(t) \int_{0}^{t} U\left(t-t^{\prime}\right) \psi_{\delta}\left(t^{\prime}\right)\left[b u^{2} \bar{u}_{x x}+c|u|^{8} u\right]\left(t^{\prime}\right) d t^{\prime}
$$

In the sequel we will prove that $S$ is well defined and it is a contraction map on $Z$. 
By Lemma 2.3 and Lemmas 2.5-2.6, for $\frac{1}{2}<b<b^{\prime}<\frac{5}{8}$, we obtain

$$
\begin{aligned}
\|S u\|_{X_{s, b}} & \leq C_{0}\left\|u_{0}\right\|_{H^{s}}+C_{1} \delta^{b^{\prime}-b}\left(\|u\|_{X_{s, b}}^{3}+\|u\|_{X_{s, b}}^{9}\right) \\
& \leq C_{0}\left\|u_{0}\right\|_{H^{s}}+C_{1} \delta^{b^{\prime}-b}\left(2^{3} C_{0}^{3}\left\|u_{0}\right\|_{H^{s}}^{3}+2^{9} C_{0}^{9}\left\|u_{0}\right\|_{H^{s}}^{9}\right) .
\end{aligned}
$$

If we take $\delta$ such that $C_{1} \delta^{b^{\prime}-b}\left(2^{3} C_{0}^{2}\left\|u_{0}\right\|_{H^{s}}^{2}+2^{9} C_{0}^{8}\left\|u_{0}\right\|_{H^{s}}^{8}\right)<1$, then $S Z \subset Z$.

Similarly, for $u_{1}, u_{2} \subset Z$, in an analogous way to the above, we can obtain

$$
\begin{aligned}
\left\|S u_{1}-S u_{2}\right\|_{X_{s, b}} & \leq C_{2} \delta^{b^{\prime}-b}\left(\left\|u_{1}\right\|_{X_{s, b}}^{2}+\left\|u_{2}\right\|_{X_{s, b}}^{2}+\left\|u_{1}\right\|_{X_{s, b}}^{8}+\left\|u_{2}\right\|_{X_{s, b}}^{8}\right)\left\|u_{1}-u_{2}\right\|_{X_{s, b}} \\
& \leq 2 C_{2} \delta^{b^{\prime}-b}\left(2^{2} C_{0}^{2}\left\|u_{0}\right\|_{H^{s}}^{2}+2^{8} C_{0}^{8}\left\|u_{0}\right\|_{H^{s}}^{8}\right)\left\|u_{1}-u_{2}\right\|_{X_{s, b}} .
\end{aligned}
$$

Furthermore, if we take $\delta$ such that $C_{2} \delta^{b^{\prime}-b}\left(2^{3} C_{0}^{2}\left\|u_{0}\right\|_{H^{s}}^{2}+2^{9} C_{0}^{8}\left\|u_{0}\right\|_{H^{s}}^{8}\right)<1$ then $S$ is a contraction mapping of $Z$ into itself. The desired result immediately follows from Banach's fixed point theorem. That means that there is a unique solution which solves the Cauchy problem (1.4) for $T<\delta$. The Lipschitz continuousness from $H^{s}(R)$ to $C\left([0, T] ; H^{s}(R)\right)$ is easily obtained from the above proof process.

\section{Competing interests}

The authors declare that they have no competing interests.

\section{Authors' contributions}

The authors declare that the study was realized in collaboration with the same responsibility. All authors read and approved the final manuscript.

\section{Author details}

'School of Mathematical Science, Shanxi University, Taiyuan, Shanxi 030006, China. ${ }^{2}$ School of Mathematics and Computer Science, Shanxi Normal University, Linfen, Shanxi 041004, China. ${ }^{3}$ School of Applied Science, Taiyuan University of Science and Technology, Taiyuan, 030024, China.

\section{Acknowledgements}

This work is supported by Natural Science of Shanxi province (No. 2013011003-2, No. 2013011002-2 and No. 2010011001-1), Natural Science Foundation of China (No. 11071149), Research Project Supported by Shanxi Scholarship Council of China (No. 2011-011)

Received: 9 January 2014 Accepted: 4 April 2014 Published: 06 May 2014

\section{References}

1. Fibich, G, Ilan, B, Papanicolaou, G: Self-focusing with fourth-order dispersion. SIAM J. Appl. Math. 62, 1437-1462 (2002)

2. Colliander, J, Keel, M, Staffilani, G, Takaoka, H, Tao, T: Global well-posedness for Schrödinger equations with derivative. SIAM J. Math. Anal. 33, 649-669 (2001)

3. Colliander, J, Keel, M, Staffilani, G, Takaoka, H, Tao, T: A refined global well-posedness result for Schrödinger equations with derivative. SIAM J. Math. Anal. 34, 64-86 (2002)

4. Bourgain, J: Fourier transform restriction phenomena for certain lattice subsets and applications to nonlinear evolution equations. I. Schrödinger equations. Geom. Funct. Anal. 3, 107-156 (1993)

5. Bourgain, J: Refinements of Strichartz' inequality and applications to 2D-NLS with critical nonlinearity. Int. Math. Res. Not. 5, 253-283 (1998)

6. Cui, SB, Guo, CH: Well-posedness of higher-order nonlinear Schrödinger equations in Sobolev spaces $H^{5}\left(R^{n}\right)$ and applications. Nonlinear Anal. 67, 687-707 (2007)

7. Kenig, CE, Ponce, G, Vega, L: Oscillatory integrals and regularity of dispersive equations. Indiana Univ. Math. J. 40, 33-69 (1991)

8. Bergh, J, Löfstöm, T: Interpolation Spaces. Springer, New York (1976)

9. Kenig, CE, Ponce, G, Vega, L: A bilinear estimate with applications to the KdV equation. J. Am. Math. Soc. 9, 573-603 (1996)

10. Kenig, CE, Ponce, G, Vega, L: The Cauchy problem for the Korteweg-de Vries equation in Sobolev spaces of negative indices. Duke Math. J. 71, 1-21 (1993)

11. Guo, CH, Cui, SB: Global existence for 2D nonlinear Schrödinger equations via high-low frequency decomposition. J. Math. Anal. Appl. 324, 882-907 (2006)

12. Huo, ZH, Jia, YL: Well-posedness for the Cauchy problem of coupled Hirota equation with low regularity data. J. Math. Anal. Appl. 322, 566-579 (2006) 
10.1186/1687-2770-2014-90

Cite this article as: Guo et al.: The local well-posedness for nonlinear fourth-order Schrödinger equation with mass-critical nonlinearity and derivative. Boundary Value Problems 2014, 2014:90

Submit your manuscript to a SpringerOpen ${ }^{\circ}$ journal and benefit from:

- Convenient online submission

- Rigorous peer review

- Immediate publication on acceptance

Open access: articles freely available online

- High visibility within the field

- Retaining the copyright to your article

Submit your next manuscript at $\boldsymbol{s p r i n g e r o p e n . c o m ~}$ 